\title{
TRANSFORMATIONALEN FÜHRUNG ALS MÖGLICHKEIT ZU EINEM NACHHALTIGEN ERFOLG BEI DEN GENERATIONEN Y UND Z
}

\author{
Balassa Éva - Nagy Tamás
}

\begin{abstract}
Abstrakt:
Arbeitgeber müssen erkennen, dass sich nicht nur die Erwartungen der Welt, der Unternehmen, sondern auch die von den Mitarbeitern verändert haben. Die Führung muss sich eine Lösung finden, wie sie für den nachhaltigen Erfolg geeignete Mitarbeiter findet. Dies ist aber eine wahre Herausforderung die eine, an die Situation,- und an die Mitarbeiter angepasste, flexible Führung verlangt. Die Führungskräfte müssen also alle Möglichkeiten ergreifen, die zum Erfolg der Unternehmen führen. In der Zukunft wird es auch nicht einfacher sein in dieser neuen Situation mit den verschiedenen Generationsansprüchen, für alle eine optimale Lösung zu finden, aber die transformationale Führung kann für die Führungskräfte eine große Unterstützung sein. Mit dieser Studie möchten wir über die transformationale Führung ein allgemeines Bild zeigen und die Korrelationen zwischen den Generationen und der transformationalen Führung zu zeigen und zu beweisen, warum das sehr wohl eine gute Methode wäre nachhaltig Erfolge zu erreichen. Aus Umfangsgründen haben wir die aktuellen Forschungsergebnisse zusammengefasst und synthetisierten die relevante Literatur.
\end{abstract}

Stichwörter: Transformationale Führung, Y- und Z-Generationen, Generationsunterschiede, MLQ, 360 -feedback, Management Audit

JEL-KOD: M12, O15, Q01

DOI: 10.33032/acr.2019.9.1.5 


\title{
TRANSFORMATIONAL LEADERSHIP AS AN OPPORTUNITY FOR SUSTAINABLE SUCCESS IN THE Y AND Z GENERATIONS
}

\begin{abstract}
:
Employers need to recognize that not only the expectations of the world, the companies, but also those of the employees have changed. The leadership has to find a solution that finds suitable employees for sustainable success. However, this is a real challenge that demands flexible management adapted to the situation and the employees. So executives need to seize every opportunity that leads to business success in the company. In the future, it will not be easier in this new situation with the different generation demands to find the optimal solution for all, but the transformational leadership can be a great support for the executives. With this study we want to show a general picture about the transformational leadership and are able to demonstrate the correlations between the generations and the transformational leadership. We would like to prove, why this is a good method to reach sustainable success. For reasons of scope, we have not made an own study, but summarized the current research results and synthesized the relevant literature.
\end{abstract}

Keywords: Transformational Leadership, Generations $Y$ and Z, Generation Differences, MLQ, 360 Feedback, Management Audit

\section{JEL CODES: M12, O15, Q01}




\section{Einfuibrung - Ziel und Bereich der Forschung}

Die Generationen sind ein sehr aktuelles Thema, seit die Generation Y die Arbeitswelt zu stauen gebracht hat. Die, bis vor kurzem mit Erfolg verwendeten Führungstheorien funktionierten auf einmal nicht mehr. Heute muss sich ein Manager für seine Mitarbeiter interessieren, er muss sie kennen um sie entsprechend motivieren zu können. Ein schlechtes Führungsverhalten führt zum Scheitern des Unternehmens und der Organisation (Finckler P., 2017). Wir möchten mit dieser Studie einen Weg zeigen wie ein Unternehmen in dieser neuen Situation nachhaltig erfolgreich bleiben kann und betonen, dass allein Methoden zum Erfolg nicht führen können. Der Schlüssel zum Erfolg besteht aus mehreren „Zutaten“- unter anderem emotionale Intelligenz, Kommunikation, Konfliktmanagement, coaching, mentoring - die die transformationale Führung unterstützt. Was bedeutet transformationale Führung? Es ist ein Führungsstil, wo Werte „umgeformt“ werden, die dann bei den Kollegen zu einer Leistungssteigerung führen. Eine optimale Möglichkeit um eine Lösung auf die neue Anforderungen der jungen Generationen zu finden, um sie für die Ziele der Unternehmen „umzugestalten“, und ein nachhaltiger Erfolg zu erreichen. Wir setzten voraus, dass die Führungskräfte über eine hohe emotionale Intelligenz und hohe Integrität verfügen.

Aus Umfangsgründen führten wir keine unabhängige Forschung zu diesem Thema durch, sondern synthetisierten nur aktuelle Forschungsergebnisse und fassten die relevante Literatur zu diesem Thema zusammen.

Erstmals möchten wir kurz die Generationen vorstellen und zeigen wie sie die Arbeitswelt beeinflusst haben.

\section{Wie verändern Generation $Y$ und $Z$ den Arbeitsmarkt?}

Die Generationen sind ein sehr aktuelles Thema, da sie die Unternehmer und die Führungskräfte zu stauen gebracht haben. Am Anfang war die Generation Y (19852000), die eine andere Sprache gesprochen hat, die den Arbeitgebern eine wahre Herausforderung bedeutet hat. Die „Jugend“ war immer anders, Konflikte waren auch immer da, aber die Lebens,- und Denkweise, die diese Generation angefangen hat, war für die Führungskräfte etwas Neues und vor allem haben die „ältere“ Generationen - Baby Boomer (1950-1965), Generation X (1966-1989)-, diese Sichten sehr schnell übernommen. Welche waren das? Die work -life Balance steht ihnen im Vordergrund, sie arbeiten nicht mehr rund um die Uhr, wollen für sich und für die Familie genug Zeit haben. Karriere ist ihnen nicht mehr so wichtig - wollen nicht mehr so viel Zeit und Energie in die Arbeit investieren und möchten auf keinen 
Fall immer erreichbar sein. Sobald sie mit der Arbeit unzufrieden sind, suchen sie eine andere. Loyalität wird also zum Fremdwort. Solange die ältere Generationen noch mehr auf die Sicherheit setzt, ein Z (ab 2000) wird nicht mehr nachdenken, verlässt seinen Job sofort wenn ihn etwas nicht passt. All das wird noch durch die Globalisierung, Digitalisierung verstärkt, die neuen sozialen Medien beeinflussen sie permanent, jeder will ein schönes, stressfreies Leben haben. Generation Y ist schon behüteter aufgewachsen, lernt aus den Fehlern der Eltern, die immer gearbeitet haben und wünscht sich eine andere Lebensart. (Balassa et.al., 2018) Sie hatten ein anderes Leben als Kinder, haben sich anders sozialisiert und entwickelt als die Generationen vorher. Sie haben Durchsetzungsvermögen, Selbstvertrauen, sind vielfältig. Ein gutes Beispiel für Ihre Freizeit: Wenn wir z.B. einen 60- Jährigen in der Freizeit anschauen, wird er wahrscheinlich gerne Kreuzrätsel lösen, ein 30-Jähriger verbringt dafür viel Zeit mit Facebook, oder im Internet. (Khera-Malik, 2014).

Auf der nächsten Grafik sind die wichtigsten Eigenschaften der derzeit arbeitenden Generationen zu sehen, wo man mit einem Blick die „gröbsten“ Unterschiede entnehmen kann. Generation $\mathrm{Z}$ - mit hellgrün gezeichnet - sticht besonders heraus, weil sie sich sogar vom Generation Y um so viel unterscheiden. Sie können sich nur schwer anpassen und leider nur durch High-Tech Geräte kommunizieren. Das macht die Lage noch komplizierter.

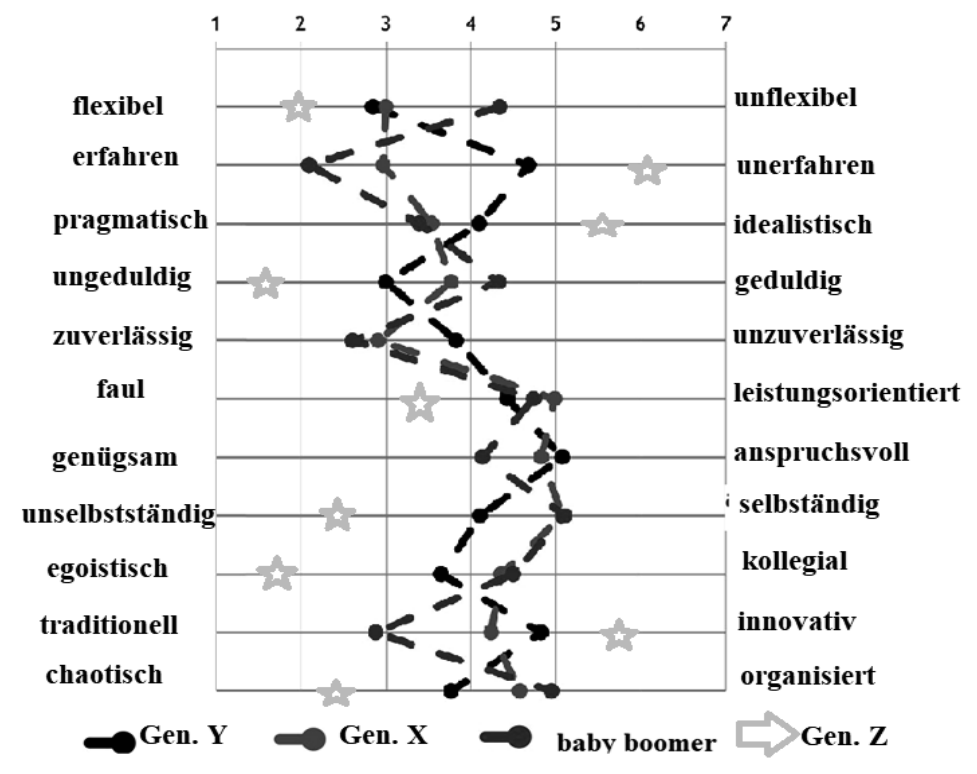

Abb.1.: Zusammenfassung der vier Generationen auf dem Arbeitsmarkt

Quelle: Eigene Bearbeitung nach der Studie von der WKO www.wko.at/.../zusammenfassung-studie-f-website-17-3-17.pdf 
Auf der zweiten Abbildung sieht man eindeutig wie sehr die Interesse und die Eigenschaften der unterschiedlichen Generationen auseinander gehen. Die baby boomer und die Generation X haben vieles gemeinsam, aber die Generation X und die Y untereinander auch. Dennoch haben die Generation Y und baby -boomer nichts miteinander gemeinsam, genauso wenig wie die drei miteinander. Das wird für die Leitung eine große Herausforderung, denn sie für den Erfolg die Generationen zueinander „schweißen“ müssen. Ganz wichtig wäre ihnen zu zeigen, dass sie voneinander lernen können, dass die älteren die Erfahrung mitbringen, die Jungen sich aber mit der digitalen Welt besser auskennen. Dafür wäre gezieltes Coaching, kontinuierliche Kommunikation, regelmäßiges Feedback und natürlich eine emotional intelligente Führung ganz wichtig.

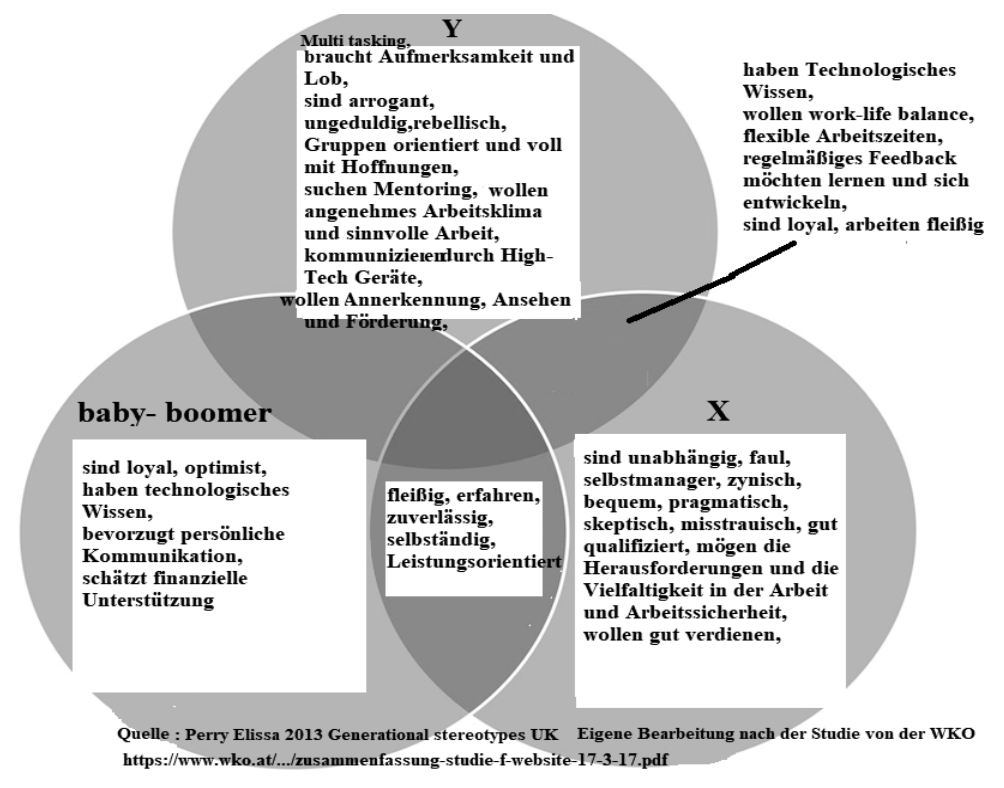

Abb.2.: Zusammenfassung der Gemeinsamkeit der vier Generationen

Quelle: Eigene Bearbeitung nach der Studie von der WKO, Perry Elissa, 2013 www.wko.at/... zusammenfassung-studie-f-website-17-3-17.pdf

Ein weiterer Unterschied gibt es auch im Einstieg in die Arbeitswelt. Ein baby - boomer (1950-1965) hatte keine Probleme bei der Job- Suche, arbeitete oft ein Leben lang in der selben Firma. Ein Teil von Ihnen wurde erst um die Wende mit Absagen konfrontiert. Ein Millenial (1985-2000) muss schon am Anfang seiner Karriere mit einer Absage rechnen und damit klarkommen. Ihnen fehlt es auch an ein ausgeglichenes wirtschaftliches und soziales Leben, was ihre Eltern gehabt haben. 
$\mathrm{Ab}$ Generation $Y$ hat sich der Arbeitsmarkt sukzessive verändert. Für die Führung und die Organisationen selber belastet diese Änderung sehr, wissen gar nicht am Anfang, wie sie damit umgehen sollen.(Woodward I.C. et al.,2015, Fogg P., 2009, Glass A., 2007) Sie sind von einer neuen Welt geprägt, stellen dem Arbeitgeber andere Wünsche und Erwartungen an. Die Kluft zwischen den alten und neuen Wertewelten scheint unüberbrückbar zu sein. Die Unternehmen müssen sich aber mit der Tatsache abfinden, dass von der alten „Welt" immer mehr in Ruhestand gehen und es bleibt ihnen nichts anderes über, als sich an diesen neuen Werten anzupassen. $\mathrm{Zu}$ niedrige Geburtsraten machen das Problem auf dem Arbeitsmarkt auch nicht besser, es entsteht ein harter Konkurrenzkampf für die Arbeiternehmer.

Mit den Z-s wird die Herausforderung nicht einfacher. Sie unterscheiden sich sogar von den Y-er so sehr, dass die zwei Altersgruppen miteinander Probleme haben. Sie sind „Digital Natives“, nehmen ihre Informationen aus verschiedenen online Quellen, kommunizieren viel zu offen online über ihr persönliches Leben, dafür haben sie im wahren Leben mit der Kommunikation große Probleme. Sie haben wenig Interesse an den anderen, Generation Z soll gescheiter sein, als Generation Y, lässt sich aber mehr beeinflussen, als Generation X. Sie haben absolut keine Loyalität zu ihrem Arbeitgeber. Sie sind hochgradig auf sich selber und auch ihre Ziele konzentriert und sind kein Teamspieler. (Generation Y ist im Vergleich sehr teamorientiert.) Freundliche Arbeitsatmosphäre, Flexibilität, work - life balance sind ihnen wichtig. Sind sehr ungeduldig und Flexibilität in der Arbeitszeit und in der Arbeitsort ist ihnen sehr wichtig. (Scholz C., 2017) Viel Kraft liegt in der individuellen Führungskultur und Motivation.

Die Generationen Y und Z brauchen eine andere flexible, erstklassige Führung, die ihre Wünsche und Erwartungen erkennt und auf diese einsteigt. Die in der Lage ist diese unterschiedliche Einstellungen und Erwartungen flexible zu führen, sie zu motivieren um die Ziele und die Werte des Unternehmens zu erreichen.

Demnächst werden wir die transformationale Führung vorstellen und zeigen wie dieser Führungsstil bei dem nachhaltigen Erfolg hilft. 


\section{Was bedeutet transformationale Führung?}

Es ist ein Konzept für einen Führungsstil bei dem die Unternehmenswerte,- und Ziele den Mitarbeitern so weitergegeben werden, dass ihr Verhalten verändert wird. (Finckler P., 2017) Die Führungskräfte versuchen z.B. mit interessanten Visionen, individuellen Entwicklungschancen und Kommunikation intrinsisch zu motivieren, treten als Vorbild auf. (Bass B.M., 1985) Die transformationale Führung ist mit der transaktionalen Führung Teil des Full Range leadership models. (Der transaktionale Führung bezeichnet einen Stil was auf einem Austauschverhältnis zwischen Manager und Mitarbeiter beruht. z. B.: Erwartungen an den Mitarbeitern, finanzielle, oder immateriellen Vorteile, Förderungen usw. (Burns J.M., 1978.)

Die Theorie zur transformationalen Führung wird von James MacGregor Burns bei Biographieanalyse von Politikern und deren Führungsstilen untersucht. Er weist auch darauf hin, dass transformationale Politiker Veränderungen bewirken konnten. Bernhard M. Bass übertrug und erweiterte diesen Gedanken auf die Mitarbeiterführung. Da die Führungskräfte eine inspirierende Vision und Identität vermitteln, entwickeln die Mitarbeiter Loyalität und Respekt der Führung gegenüber. (Bass B.M., 1985) Einen wesentlichen Beitrag zur Anwendung dieser Führung haben Bernhard M. Bass und Bruce Avolio geleistet als sie ihr Konzept mit einem Fragenbogen operationalisiert und dann auf Praxistauglichkeit empirisch getestet haben. (vgl. Bass / Avolio, 1994, S.2) Die Fragen der MLQ ( multifactor leadership questionnaire) messen mit neun Skalen fünf Aspekte über die transformationale Führung. (Bass, B.M.- Avolio, B.J., 2000). Das TLI ( Transformational leadership Inventory) mit sieben Skalen messen schon sechs Dimensionen der transformationalen Führung. (Podsakoff, P. M., MacKenzie, et.al., 1996). Die Ergebnisse können im Verhaltensinterview oder im 360 Grad- Feedback eingesetzt werden. (vgl. Pelz W., 2014) Die Ergebnisse werden dann in einem Bericht zusammengefasst, so ist eine Reflexion des eigenen Führungsverhaltens möglich. Dieser Verhalten verändernde Führungsstil hat zu so vielen positiven Ergebnissen geführt, dass sie sowohl in der akademischen Welt als auch in der Praxis verbreitet ist. (vgl. Pelz W., 2013)

Der wichtigste Kern der Methode ist der Vorbildfunktion der Führungskraft. Sie schafft Vertrauen, wirkt inspirierend auf die Mitarbeiter, muss deren Selbständigkeit erreichen und eine offene Kommunikation führen. Abbildung 1. zeigt das Prinzip und den Zusammenhang zwischen dem Verhalten von Managern und Mitarbeitern und die Bereichen auf die der Stil direkt wirkt. (Pelz W., 2016) 


\section{Das Prinzip der Transformationalen Führung}

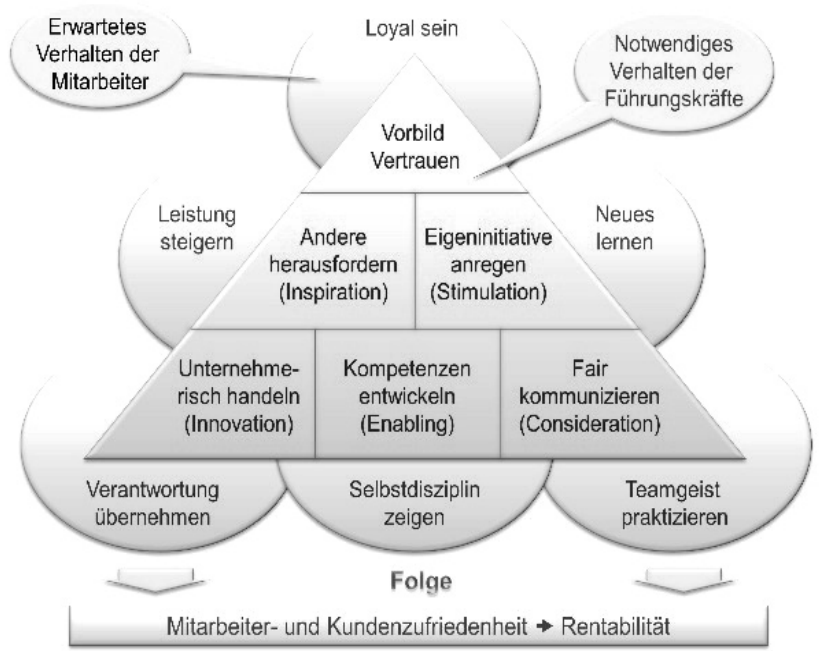

(C) Institut für Management-Innovation - Prof. Dr. Waldemar Pelz

Abb.3. : Das Prinzip in der Transformationalen Führung

Quelle: Prof. Dr. Waldemar Pelz, (2016) Transformationale Führung - Forschungsstand und Umsetzung in der Praxis, S. 95

Dr. Pelz hat mit dieser Grafik die wichtigsten Elemente über das Verhalten der Mitarbeiter und der Führung zusammengestellt, die durch die Mitarbeiter und die Kundenzufriedenheit zum Erfolg führen. Die einzelnen Bereiche geben den Mitarbeitern die Möglichkeit um sich weiterzuentwickeln und zeigt ein sehr durchgedachtes, miteinander verbundetes System.

Die Forschungsergebnisse vom Dr. Pelz sind aus dem Jahr 2009-2010 und basieren auf 14348 Befragungen unter Fach-und Führungskräften. (Pelz W., 2010)

\section{Bass ordnet die Verhaltensweisen der Manager in vier Kategorien zu:}

1. Vorbildfunktion: Die Führungskraft ist integer, glaubwürdig, gilt als Vorbild - hat hohe ethische- und moralische Standards. Die Manager bevorzugen das Organisationsinteresse ihre persönlichen Ziele und Vorteile.

2. Inspirierende Motivation: mit einer inspirierenden Vision wird die intrinsische Motivation der Mitarbeiter gesteigert. Führungskräfte vermitteln Sinn und Bedeutung, wofür es für die Mitarbeiter sich lohnt Zeit und Energie zu investieren. 
Die ersten zwei werden oft als Charisma einer Führungsperson bezeichnet, dessen Wirkung natürlich auf die Mitarbeiter sich sehr positiv ist.

3. Intellektuelle Anregung: Die Führungsperson versucht die kreativen und die innovativen Fähigkeiten des Kollegen so zu fördern, dass sie sich herausgefordert fühlen und in der Lage sind Unternehmensprozesse hinter zu fragen bzw. zu optimieren.

4. Individuelle Unterstützung: Chefs werden nach den individuellen Bedürfnisse der Kollegen helfen z.B.: als Mentor, coach, Konsulent etc. damit sie gezielt deren Fähigkeiten und Stärken fördern können. Dabei wecken sie neue Motive und Bedürfnisse, unterstützen ihre Selbständigkeit und Selbstvertrauen. (Bass B.M., Avolio B.J., 1990)

Die wichtigsten Erkenntnisse aus vielen Studien zeigen eindeutig, dass transformational geführte Mitarbeiter zufriedener, motivierter, kreativer, verantwortungsbewusster sind als die transaktional geführten. Der Stil hat auf die Führungskräfte auch einen großen Einfluss: haben gute Beziehungen zu den Kollegen, haben mehr Energie, sind wesentlich leistungsfähiger und stress resistenter. (Geyer A.L, Steyrer J.M., 1998; Pelz W., 2016, S.97.)

\section{Die Nachbaltigkeit der transformationalen Fübrung}

Die Nachhaltigkeit dieser Führungsmethode basiert auf der Validierung des Fragebogens, auf die empirischen Belege über die Wirksamkeit in der Praxis, auf dem erfahrenen wirtschaftlichen Erfolg und auf die beobachteten veränderten Verhaltensweisen. (Pelz W., 2016)

\section{Kritik dieser Fübrung}

$\mathrm{Zu}$ den Schwächen der Stil gehören die Vernachlässigung mancher beim Erfolg wichtige Kompetenzen, die Mehrdeutigkeit mancher Grundbegriffe, die Betonung heroischer Aspekte (Yukl A., 2013). Entscheidend wäre, dass die Führungskräfte den Unterschied zwischen den transformationalen- und den transaktionalen Führung verstehen. So ist z.B. vermeidbar, dass sie nur belohnen oder bestrafen, denn transformational zu führen ist viel mehr als das. Transformationale Führung konzentriert sich mehr auf den Menschen und probiert mit coaching zu erreichen, was für die Zukunftsziele der Organisation wichtig wäre. Dennoch ist es sehr wichtig, dass die 
Führungskräfte die Kollegen bei der Arbeit kontinuierlich unterstützen sich in den Abläufe und Probleme „einmischen“, denn ohne die Richtlinien zu zeigen, kann dazu führen, dass der ersehnte Erfolg ausbleibt. Ganz ohne Kontrolle geht also nicht, dennoch muss man die richtige „Art“ und „Menge“ von der Kontrolle aussuchen. Die Führungskräfte neigen sich also in der Praxis zu einem „Mischform“ des Stiles. (Eyring A., 2017) Das ist aber verständlich, da es genug tagtägliche Aufgaben gibt, wo Charisma nicht genug ist für die Umsetzung eines Zieles. (Pelz W., 2016)

Wichtig sind, dass sie tatsächliche, sichtbare Werte der Organisation merkbar und „spürbar“ sind. Darauf weisen (Burns J. 2003, Schumpeter A. 1950, Gardner H. 1995, Hoffman B. 2011) hin. Außerdem müssen wir die Wichtigkeit von der Kommunikation betonen. Bei den Fragebögen muss man sich darauf achten, dass man die „richtigen“ Fragen stellt, transformationale- und transaktionale Verhaltensbeschreibungen gehören getrennt.

\section{Umsetzung der transformationale Fübrung}

Mit klaren Werte- und Verhaltenserwartungen an die Mitarbeiter und mit ständigen Umsetzungskontrollen ist die kulturelle Veränderung möglich. Wichtig sind einfache, für jeden leicht verständliche Ziele, Glaubwürdigkeit, eine offene Kommunikation zwischen Führung und Kollegen. Ebenso bedeutsam ist ein permanentes Feedback über die Leistung, oder über die Änderungen. Oft scheitert der Praxis daran, dass die Ziele und die Führungsgrundsätze zu abstrakt formuliert und nicht validiert wurden. Wenn man mit einem validierten Diagnoseverfahren anfängt, kann es auch für Mitarbeitern oder für Führungskräfte- für eine Selbsteinschätzung, ein $360^{\bigotimes}$ -feedback, oder ein Verhaltensinterview verwendet werden. Dieses „soll- ist“ Vergleich ermöglicht den Fehler zu korrigieren und sich weiterzuentwickeln. (Pelz W., 2014) Die Verbesserungsvorschläge werden zu einem individuellen, persönlichen Entwicklungsplan, den der Mitarbeiter mit dem Vorgesetzten bespricht. Im ersten Teil wird an der Verhaltensänderungen gearbeitet - z.B.: klare Kommunikation der Werte, oder Ziele. Im zweiten Teil kommt eine Entwicklungsplan für die Zukunft besprochen -z.B.: welche Kompetenzen - gefördert werden sollen. Nur durch die Verhaltensänderung von Mitarbeiter ist es möglich einen Verbesserungsprozess zu erzielen, sowohl bei den Mitarbeiter, als auch bei den Führungskräften. (Pelz W., 2014 )

Durch die Ergebnisse des Fragebogens bekommt man wertvolle Informationen, Eigenschaften und Kompetenzen über die Arbeitskraft, die ihre Eigenschaften und Wünsche verrät. Der Manager muss „nur“ mit der Hilfe dieser Präferenzen und Motivationen die Einstellung „verändern“. Mit der Änderung wird es möglich ihre 
Denkweise zu ändern, die Unternehmenswerte,- und Ziele verständlich zu machen und ihre Zukunftspläne zu erforschen und so die Mitarbeiter in eine gewünschte Richtung gezielt „umwandeln“-.

\section{Ergebnisse: die Korrelationen zwischen Generationen und die transformationa- le Führung}

Hier möchten wir die Korrelationen zwischen die Generationen und der transformationale Führung zeigen und beweisen, warum das sehr wohl eine gute nachhaltige Methode wäre für die Organisationsentwicklung der unterschiedlichen Generationen.

Die Generation Y und Z brauchen die Aufmerksamkeit und die Anerkennung der Führung. Da sie wenig bis gar keine Kritik vertragen, müssen die Arbeitgeber zu anderen Mitteln greifen. Die Abbildung 1. vom Dr. Pelz umfasst Gebiete, wo die jungen Generation von den älteren sich sehr unterscheiden und wo man sie mit kleineren Schritten „umformen“ oder „anpassen“ sollte, die positiv auf die Zusammenarbeit von den verschiedenen Generationen auswirken würde. Die Wünsche zu erfüllen ist es auch nur möglich, wenn man sie und ihre Vorstellungen kennt. Diese Praktiken „liefern“ genug Informationen über die Kompetenzen, Wünschen, Vorstellungen der Mitarbeiter, womit die Manager sie kennenlernen können. Ein Gespräch mit dem Chef während des Ausfüllens des Fragenbogens steigert ihr „Wichtigkeits“Gefühl und kann Ihre Loyalität stärken. Die gute Kommunikation hilft auch bei den ständigen „Feedbacks“ die die Millenials permanent brauchen. Das umgekehrt stärkt die Organisation selber, da jede Art von Problemen sofort ansprechbar und lösbar ist. Das bringt also für das Konfliktmanagement viel, da eine Generation Z die persönliche Art von Kommunikation lernen könnte, da diese Altersgruppe durch das Online - Kommunizieren Mängel aufweist. All die über die Mitarbeiter erhaltene Informationen können in die Verhaltensveränderungsprozess eingebaut werden und sind für die Organisationskultur sehr nützlich.

Da die neuen Generationen eine große Wert auf den Sinn in der Arbeit legen, gäbe es die Möglichkeit ihnen einen interessanten Arbeitsbereich zu zeigen und das bei einem Chef, der sich vorbildlich benimmt. Dank der transformationalen Führung hat der Manager „einen Schlüssel“ in der Hand, da er seine Kollegen und dessen Stärken, Schwächen, Zukunftspläne kennt, somit kann er leichter ihnen die für sie geeignete Arbeit zuordnen. Somit könnte ihre Motivation gesteigert werden und das Unternehmen wäre einen Schritt näher um ihre Loyalität zu gewinnen. Das wirkt auf das Arbeitsklima auch aus. Die Informationen der Fragebogen helfen die Zufriedenheit der Mitarbeiter zu den Zielen zu begleiten, bzw. „manipulieren“. 
Diese Entwicklungsarbeit kann man mit Training und coaching unterstützen. Als Kontrolle und Feedback kann ein Management - Audit erfolgen.

Ein gutes Argument sich mehr mit den Mitarbeitern zu beschäftigen ist auch Folgendes:

Da die Menschen einen großen Teil ihres Lebens mit der Arbeit verbringen, trägt es viel zur ihrer Identitätsentwicklung bei. Aus diesem Grund muss es auch im Interesse der Arbeitgeber stehen, dass sie sich hier wohlfühlen. (Gyökér et al., 2016) Spector meint, dass die hohe Mitarbeiterzufriedenheit ein Indikator für die emotionale und psychische Gesundheit der Menschen und der Organisation wäre. Die Kollegen, die mit ihrer Arbeit zufrieden sind, sind in ihrem Privatleben ausgeglichener und können konzentrierter, effektiver und motivierter arbeiten. Der Weg zum Erfolg besteht aus der einzelnen Leistung der Mitarbeiter. Das verstärkt die Tatsache, dass die über die Mitarbeiter erforschte Informationen richtig umgesetzt zu einem erfolgreichen Unternehmen führen können. (Spector E.P., 1997)

Bei Abbildung 1. sieht man diese Zusammenhänge zwischen den Erwartungen von den Angestellten und dem Verhalten der Führung. Auf der Mitarbeiter Seite sieht man Erwartungen, wo sich die neuen Generationen unbedingt bessern sollten, wie Verantwortung zu übernehmen, Leistung zu steigern, Loyalität oder etwas Neues zu lernen. Die Manager Seite setzt eine hohe emotionale Intelligenz aus, um überhaupt fähig zu sein sich neben den Führungsaufgaben auf die Mitarbeiter konzentrieren zu können. Ein Vorbild zu sein ist bestimmt eine gute Voraussetzung. Das Modell fasst Grundsätze zusammen, die der Mitarbeiter,- und Führungsentwicklung fördern und somit für die Organisation eine kontinuierliche Werdegang ermöglichen. Diese große Fürsorglichkeit der Mitarbeiter wodurch ihre Wünsche und Vorstellungen genauso wichtig sind, wie die von den Kunden, hat schon öfters zum Erfolg geführt. Frederick Smith, der Gründner von FedEx meinte auch, dass sein Erfolg ist auf die Mitarbeiterzufriedenheit zurück zu führen, denn nur das bringt die erwünschte Kundenzufriedenheit mit sich. Zemke - Raines - Filipczak haben Forschungen bei fünf großen deutschen Unternehmen durchführt und behandelten die Arbeitnehmer als wären sie Kunden. Sie haben ihre Präferenzen erforscht, hatten Freiheiten in der Zeitraum und in der Ort der Arbeit, aber mit der Arbeit müssten sie rechtzeitig fertig sein. Bei der Teamarbeit haben sie Menschen in eine Gruppe zusammen arbeiten lassen, die zueinander gepasst haben und so erreichten sie lange und große Erfolge. All das wurde noch durch mentoring und coaching unterstützt und der Erfolg dieser Firmen war riesig. (Zemke et. al. (2013) Auch in diesen Forschungsergebnissen haben die Interesse und die Kompetenzen der Mitarbeiter eine wichtige Rolle gespielt und führten zum Erfolg. Die Mitarbeiter und ihre Wünsche zu erforschen steht bei der transformationalen Führung auch im Mittelpunkt. Durch die Ergebnisse dieser Forschungen haben wir eine weitere Bestätigung dafür, dass man die Mitarbeiter und ihre Ansprüche ernst nehmen muss. Man merkt, wie 
wichtig die Rolle den Kollegen auf dem Weg zum Erfolg ist und was alles möglich ist, wenn sie in einem guten Arbeitsklima gerne und motiviert arbeiten. Richtig zu führen ist bei Generationen Y und Z sowieso eine Kunst, dennoch nicht unmöglich, wenn man Ihre Vorstellungen kennt und denen nachgeht.

\section{Die Wirkung der transformationalen Fübrung}

Die Forschungsergebnisse vom Dr. Pelz zeigen, dass dank dieser Führungsstil die Mitarbeiter mit mehr Kreativität gearbeitet haben, mehr Leistung gebracht haben, ihre intrinsische Motivation und die Arbeitszufriedenheit gestiegen ist. Wichtig sind bei der Methode eindeutige, anspruchsvolle Ziele, die durch eine offen kommunizierende Führungsperson vermittelt werden. Die Aufgaben, die zu den Kompetenzen der Mitarbeiter passen, stärken das Selbstvertrauen und die Verantwortung der Kollegen. Von der Manager - Seite, dank der transformationale Führung haben die Führungskräfte eine bessere Beziehung zu den Mitarbeitern, hatten weniger Stress, dafür mehr Energie in der Arbeit. Dadurch entsteht ein nettes Arbeitsklima, wo jeder gerne arbeitet und das ist den Generation $\mathrm{Y}$ und $\mathrm{Z}$ sehr wichtig. Diese Art von Führung ist eine auf Grundinformationen basierende, konsequente Weiterentwicklung mit Zielvereinbarung, die durch ein Management - Audit eine verlässliche Kontrolle ermöglicht.

\section{Konklusion}

Der Arbeitsmarkt hat sich seit der Generation Y verändert, das Angebot der Arbeitskräfte wird immer knapper. Die Führung muss sich eine Lösung finden, wie sie für den nachhaltigen Erfolg geeignete Mitarbeiter findet. Dies ist aber eine wahre Herausforderung die eine, an die Situation,- und an die Mitarbeiter angepasste, flexible Führung verlangen.

Wir sind der Meinung, dass eine neue flexible Unternehmenskultur mit kompetenten, interessierten, sich vorbildlich benehmenden Führungskräften, die bereit sind gegenüber den Arbeitnehmern offen und interessiert zu sein, also auch über eine hohe emotionale Intelligenz verfügen, mit ihrem Verhalten viel zum Erfolg dazu beitragen. Unternehmer und Führungskräfte muss es Bewusst sein, dass Generation Z absolut keine Loyalität gegenüber den Mitarbeitern oder dem Arbeitgeber hat. Sobald etwas ihren Erwartungen nicht entspricht, wechseln sie ihre Arbeit. In der Arbeit suchen sie die Sinnhaftigkeit und die Erweiterung ihrer Kompetenz. Aus diesem Grund ist die transformationale Führung eine optimale Möglichkeit, weil man 
sich durch die Ergebnisse der MLQ auf ihre Wünsche einstellen kann, sie werden ernst genommen und sie können sich auf den verschiedensten Bereichen entwickeln. Die positive Arbeitsklima wirkt auch sehr anziehend. Die Führungsperson muss aber den Übersicht behalten und sich fürs Erreichen der gewünschten Ziele einsetzen. Wir sind uns sicher, dass auch diese Generation gerne zu einem erfolgreichen, dynamischen Team gehört, das durch starke Werte verbunden ist, nur man muss ihren Prioritäten akzeptieren und den Weg finden sie in die gewünschte Richtung zu „formen“. (Scholz C., 2014, Balassa E.et. al. 2018)

Bei den herausfordernden Situation der jetzigen Arbeitswelt muss eine Führungskraft alle Möglichkeiten ergreifen, die zum Erfolg der Unternehmen führen. In der Zukunft wird es auch nicht einfacher sein in dieser neuen Situation mit den verschiedenen Generationsansprüchen, für alle eine optimale Lösung zu finden, aber die transformationale Führung kann für die Führungskräfte eine große Unterstützung sein.

Diese Studie ermöglicht über die tranformationale Führung ein allgemeines Bild zu bekommen. Wir haben das Thema in Verbindung mit den Herausforderungen der jungen Generationen vorgestellt und systematisiert. Unser Ziel war die Korrelationen zwischen die Generationen und der transformationalen Führung zu zeigen und zu beweisen, warum das sehr wohl eine gute Methode wäre nachhaltig Erfolge zu erreichen. Aus Umfangsgründen führten wir keine unabhängige Forschung zum Thema durch, sondern synthetisierten nur aktuelle Forschungsergebnisse und fassten die relevante Literatur zu diesem Thema zusammen.

\section{Literatur:}

[1.] Bass, B. M. (1985): Leadership and performance beyond expectations, New York, Free Press, pp.191

[2.] Bass, B. M., Avolio, B. (1994). Improving organizational effectiveness through transformational Leadership, Thousand Oaks, Sage Publication, pp. 248

[3.] Bass, B. M., Avolio, B. J. (1997). Full range leadership development manual for the multifactor leadership questionnaire, Palo Alto, Mindgarden Inc., pp. 170

[4.] Bass, G. (2008). The Bass Handbook of Leadership. Theory, Research, and Managerial Applications, New York, Free Press, pp. 1536

[5.] Bass, B. M., \& Avolio, B. J. (2000), MLQ: Multifactor leadership questionnaire, Redwood City, CA, Mind Garden 
[6.] Burns J.M. (1978) Leadership, New York, Harper \& Row , pp. 530

[7.] Burns, J. M. (2003) Transforming Leadership, New York, Grove Press, pp.320

[8.] Eyring A. (2017) Pacing for Growth, Oakland, Berrett-Koehler Pblishers Inc., pp. 183

[9.] Finckler P. (2017), Transformationale Führung, Wegweiser für nachhaltigen Führungs - und Unternehmenserfolg, Berlin Heidelberg, Springer-Verlag, pp. 256

[10.] Hesse G. - Mattmüller R. ( 2015) Perspektivwechsel im Employer Branding, Neue Ansätze für die Generationen Y und Z, Berlin Charlottenburg, Springer Gabler, pp. 208

[11.] Klaffke Martin (2014) Generation Management: Konzepte, Instrumente, Good -Practice- Ansätze, Wiesbaden, Springer Gabler, S.260,

[12.] Scholz Christian, Generation Z: Wie sie tickt, was sie verändert und warum sie uns alle ansteckt, Weinheim, Wiley-VCH, pp.220

[13.] Scholz Christian (2017), Generation Z als Herausforderung für die Berufschule, Bozen, Verlag Retina, S.244.,

[14.] Spector E.P. (1997), Job Satisfaction, application, assessment, causes and consequences, Soth Florida, Sage Publ.Inc. , pp.96

[15.] Schumpeter J.A. (1950) Kapitalismus, Sozialismus und Demokratie 6.Aufl.1987, Tübingen, Francke Verlag, pp. 542

[16.] Zemke R.-Raines C.-Filipczak B. (2013)Generations at Work: Managing the Clash of Boomers, Gen Xers, and Gen Yers in the Workplace, American Management Association, 312pp.

[17.] Yukl A., (2013) Leadership in Organization, 8. Aulf., New York Harlow, Pearson, pp. 528

\section{Buchausschnitt:}

[18.] Avolio, B. J. \& Bass, B. M. (1994) The full range of leadership development programs, Basic and advanced manuals, Binghamton, 1-28

[19.] Dr. Gyökér Irén (2016), Dr. Finna Henrietta, Krajcsák Zoltán, Daruka Eszter, Dr. Szabó Tibor: Szervezeti viselkedés (Oktatási segédanyag), Budapest, 41-63.old. 
[20.] Kertzer, D. I.(1983) Generation as a sociological problem, Annual Review of Sociology, No. 9., p. 125-149.

[21.] Khera S.N.- Malik S. (2014), New Generation-great expectations exploring the work attributes of gen. Y, Philadelpia, Global Journal of Finance and Management, Volume 6, Number 5 pp. 433-438.

[22.] Pelz, W. (2016): Transformationale Führung - Forschungsstand und Umsetzung in der Praxis. In: Leadership und angewandte Psychologie. Band 1: Wirksame und nachhaltige Führungsansätze. Berlin, Springer Verlag 2016, pp. 93-112

[23.] Podsakoff, P. M., MacKenzie, S. B., \& Bommer, W. H. (1996) Transformational leader behaviors and substitutes for leadership as determinants of employee satisfaction, commitment, trust, and organizational citizenship behaviors In: Journal of Management 22 (2), Waschington, S. 259-298.

\section{Zeitschrift:}

[24.] Balassa É. - Kópházi A. (2018) Wie verändern Generation Y und Z den Arbeitsmarkt? Pécs, Tudásmenedzsment 19/2, pp. 78-85

[25.] Balassa É. - Kópházi A. - Pétervári Zs. (2018) Az X, Y és Z generációk kihívásai a 21. század munkaerőpiacán, Budapest, Munkaügyi Szemle 18 (6), pp. $49-55$

[26.] Bass, B. M., \& Avolio B. J. (1990): Developing Transformational Leadership: 1992 and Beyond. Journal of European Industrial Training, 14 (5), $21-27$.

[27.] Fogg, P. (2009): When generations collide. Education Digest: Essential Readings Condensed for Quick Review, Pennsylvania, p.25-30.

[28.] Geyer, A. L. J. \& Steyrer, J. M. (1998): Messung und Erfolgswirksamkeit transformationaler Führung, Zeitschrift für Personalforschung 12, München, 377-401.

[29.] Glass, A. (2007): Understanding generational differences for competitive success. Industrial and Commercial Training, Pennsylvania, p. 98-103.

[30.] Hoffman, B. J., Bynum, B. H., Piccolo, R. F., Sutton, A. W., (2011). Person-Organization Congruence: How Transformational Leaders Influence Work Group Effectiveness? Georgia, Academy of Management Journal 54, 779-796 
[31.] Joshi, A. - Dencker, J. C. - Franz, G.(2011) Generations in organizations, Research in Organizational Behavior, USA, No. 31., p. 177-205.,

[32.] Pelz W. (2010) Fokussieren statt verzetteln (Volition als Umsetzungskompetenz), in: Personal, Zeitschrift für Human Resource management, Düssedorf, Nr. 4/2010, pp. 30-32

[33.] Pelz W. (2013) Auf die Probe gestellt, Personalmagazin 1/2013, Freiburg im Breisgau, pp. 36-38.

[34.] Pelz W. (2014) Das 360-Grad Feedback zur Erkennung und Entwicklung von Potentialträgern. In: In Deutschland führen die Falschen. Wie sich Unternehmen ändern müssen. Berlin, Helios Media, pp. 251-285

[35.] Woodward, I. C. - Vongswadi, P. -More, E. A. (2015): Generational diversity at work: a systematic review of the research, Working Paper Series, Australia, INSEAD Business School, pp.72.

\section{Internet:}

Zusammenfassung der vier Generationen auf dem Arbeitsmarkt (2017) www.wko. at/../zusammenfassung-studie-f-website-17-3-17.pdf , hinuntergeladen: 01.12.2018 09:12

\section{Verfasser:}

\section{Balassa Éva}

Doktorand an der Széchenyi István Doktoratschule, Fakultät für Management und Betriebswirtschaft,

Gasthof Kirchenwirt

E-mail: evi.balassa@gmail.com

\section{Nagy Tamás}

Doktorand an der Széchenyi István Doktoratschule, Fakultät für Management und Betriebswirtschaft, Unternehmensberater Email: nagy.tamas801@gmail.com 